\title{
Post-disaster Surveillance among State Health Departments in the United States
}

\author{
Erin Simms*2, Amy Wolkin'1, Ekta Choudhary ${ }^{1}$, Robert Mathes ${ }^{3}$, Michael Heumann² and \\ Sharon Watkins ${ }^{4}$
}

${ }^{1}$ Centers for Disease Control and Prevention, Atlanta, GA, USA; ${ }^{2}$ Council of State and Territorial Epidemiologists, Atlanta, GA, USA;

${ }^{3} \mathrm{New}$ York City Department of Health and Mental Hygiene, New York, NY, USA; ${ }^{4}$ Florida Department of Health, Tallahassee, FL, USA

\section{Objective}

The panel will discuss the current status of disaster surveillance capabilities at local and state health departments in the United States and will provide an overview of current resources available to epidemiologists for surveillance.

\section{Introduction}

The role of public health in preparing for, responding to, and recovering from emergencies has expanded as a result of the massive impact recent disasters have had on affected populations. Nearly every large-scale disaster carries substantial public health risk and requires a response that addresses immediate effects of the disaster on a population (e.g., mass casualties and severe injuries, lack of shelter in severe weather), as well as subsequent secondary physical effects (e.g., carbon monoxide poisoning due to improper operation or location of carbon monoxide-producing devices such as generators) and emotional effects (e.g., grief, anxiety, and post-traumatic stress disorder) caused by the disaster. Disaster epidemiology has been identified as an evolving field that integrates a variety of data sources and technological and geospatial resources to expedite reporting and to increase the accuracy of information collected and used by emergency planners and incident managers.

As the national organization that supports the activities of applied epidemiologists in state, tribal, local, territorial, and federal public health agencies, the Council of State and Territorial Epidemiologists (CSTE) assembled a Disaster Epidemiology Subcommittee of public health experts and practitioners from diverse fields of applied epidemiology to discuss the use of epidemiologic methods in all phases of the disaster management cycle. In 2012, the Subcommittee assessed state-level disaster epidemiology capacity with a focus on surveillance. Concurrently, the Centers for Disease Control and Prevention (CDC) created the Disaster Epidemiology Community of Practice (DE CoP), which serves as a forum for epidemiologists and other public health practitioners working in the applied setting to share information, including resources related to post-disaster surveillance. This panel will present recent findings, experiences, and recommendations of the Disaster Epidemiology Subcommittee and the DE CoP.

\section{Methods}

The panel will consist of three 20-minute talks:

1) Overview of surveillance in disaster settings and results from CSTE's 2012 assessment of state-level disaster epidemiologic capacity, with specific focus on surveillance capabilities (Amy Wolkin, CDC)

2) Emerging issues in disaster surveillance, including utilization of syndromic surveillance and the Environmental Public Health Tracking Network (Michael Heumann, CSTE Consultant; Robert Mathes, NYC DHMH)

3) New applications of surveillance and Post-disaster surveillance in Florida: A state health department's perspective (Sharon Watkins, FL DOH; Ekta Choudhary, CDC)
Each talk will be followed by five minutes of discussion, with 20 minutes for closing comments on the future directions of disaster surveillance practices.

\section{Results}

Areas of discussion that may arise include: limitations of and best practices for modifying an existing surveillance system for use in the disaster setting versus development of a separate surveillance system; ways in which utilization of and involvement in the DE CoP can be optimized for public health practitioners at the state and local level; collaboration with emergency management personnel to maximize efficiency of surveillance and other epidemiologic activities; and future roles and directions for use of other epidemiologic activities (e.g., rapid needs assessments) in preparing for and responding to emergencies.

\section{Conclusions}

This panel is intended to facilitate discussion on current disaster surveillance practices and to inform the audience about available tools and resources to assist preparedness and response activities in their jurisdiction. It is also intended that this panel may serve as a forum for the audience to share their experiences in order to generate further ideas on how disaster surveillance practices might be further improved.

\section{Keywords}

Surveillance; Disaster; Disaster epidemiology

\section{Acknowledgments}

The authors would like to acknowledge the substantial contributions of members of the CSTE Disaster Epidemiology Subcommittee, CDC Disaster Epidemiology Community of Practice, and state assessment respondents all of whom have contributed to improvement of disaster epidemiology surveillance.

\section{*Erin Simms}

E-mail: esimms@cste.org 